\title{
Commentary
}

\section{COVID-19 in the Shadows of MERS-CoV in the Kingdom of Saudi Arabia}

\author{
Mazin Barry ${ }^{1, \dagger}$, Maha Al Amri ${ }^{2, \dagger}$, Ziad A. Memish ${ }^{3,4,5, *}$ \\ ${ }^{1}$ Division of Infectious Disease, College of Medicine, King Saud University, Riyadh, Kingdom of Saudi Arabia \\ ${ }^{2}$ Department of Infectious Disease, King Faisal Specialist Hospital and Research Center, Riyadh, Kingdom of Saudi Arabia \\ ${ }^{3}$ Director Research and Innovation Centre, King Saud Medical City, Ministry of Health, Riyadh, Kingdom of Saudi Arabia \\ ${ }^{4}$ College of Medicine, Alfaisal University, Riyadh, Kingdom of Saudi Arabia \\ ${ }^{5}$ Hubert Department of Global Health, Rollins School of Public Health, Emory University, Atlanta, GA, USA
}

\section{ARTICLE INFO}

\section{Article History}

Received 16 February 2020

Accepted 16 February 2020

Keywords

SARS-CoV-2

MERS-CoV

COVID-19

Saudi Arabia
Middle East Respiratory Syndrome Coronavirus (MERS-CoV) has plagued the Middle East since it was first reported in 2012. Recently, at the end of December 2019, a cluster of pneumonia cases were reported from Wuhan city, Hubei Province, China, linked to a wet seafood market with a new coronavirus identified as the etiologic agent currently named SARS-CoV-2. Most cases are in Mainland China with international spread to 25 countries. The novelty of the virus, the rapid national and international spread, and the lack of therapeutic and preventative strategies have led the WHO International Health Regulation emergency committee to declare the disease as Public Health Emergency of International Concern (PHEIC) on January 30, 2020. As it relates to countries with the ongoing MERS-CoV community cases and hospital acquired infections, there will be a huge challenge for HCWs to deal with both coronaviruses, especially with the lack of standardized and approved point of care testing. This challenge will now be faced by the whole global health community dealing with COVID-19 since both coronaviruses have similar presentation. Those patients should now be tested for both MERS-CoV and SARS-CoV-2 simultaneously, and with the continuing wide international spread of SARS-CoV-2, the travel history to China in the last 14 days will be of less significance

(C) 2020 The Authors. Published by Atlantis Press International B.V. This is an open access article distributed under the CC BY-NC 4.0 license (http://creativecommons.org/licenses/by-nc/4.0/).

\section{INTRODUCTION}

Middle East Respiratory Syndrome Coronavirus (MERS-CoV) has plagued the Middle East since it was first reported in 2012 [1]. As of January 2020, the World Health Organization (WHO) has been notified of 2494 laboratory-confirmed cases with 858 mortalities [2], with the majority of cases reported from the Kingdom of Saudi Arabia (KSA), which-at the time of this writing-has a total of 2131 laboratory-confirmed cases with 827 deaths [3], making the case fatality rate (CFR) $39 \%$. Currently, KSA continues to report MERS-CoV cases on weekly basis, but the overall number of nosocomial transmissions has significantly been reduced [4-7]. Recently, at the end of December 2019, a cluster of pneumonia cases were reported from Wuhan city, Hubei Province, China, linked to a wet seafood market with a new coronavirus identified as the etiologic agent currently named Severe Acute Respiratory Syndrome (SARS-CoV-2) $[8,9]$. To date, the virus has rapidly spread causing - at the time of this writing - a total of 50,272 laboratory-confirmed cases and 16,522 clinical cases (total cases 66,794) with 1524 deaths (CFR 3\%). Most cases are in Mainland China $(66,576)$, with 25 other countries affected [10] (Figure 1, adapted from WHO) including eight cases in the United Arab Emirates. The novelty of the virus, the rapid national and international spread, and the lack of therapeutic and preventative strategies have led the WHO

"Corresponding author.Email: zmemish@yahoo.com

${ }^{\dagger}$ Additional information: MB, mbarry@ksu.edu.sa; MAA, malamari70@kfshrc.edu.sa
International Health Regulation (IHR) emergency committee to declare the disease as Public Health Emergency of International Concern (PHEIC) on January 30, 2020. According to the latest China Corona Virus Disease (CDC) update, COVID-19 suspected case definition needs to fulfill an epidemiological criterion and at least two of the clinical criteria, and in the absence of an epidemiological link, fulfill all clinical criteria. Confirmed case is a suspected case with a laboratory confirmation. Epidemiological and clinical criteria are highlighted in Table 1.

The Global Surveillance Interim guidance by WHO developed [11] the following case definition for suspect case: a patient fulfilling either criteria A or B as detailed in Table 2.

In contrast, the Saudi Center for Disease Prevention and Control (SCDC) defined a suspected case as the following [12]:

A person with acute respiratory illness (ARI) (fever with cough and/or shortness of breath) AND any of the following:

1. A history of travel to China in the 14 days prior to symptom onset.

2. A close physical contact in the past 14 days with a confirmed case of COVID-19 infection.

As it relates to KSA and with the ongoing MERS-CoV community cases and hospital acquired infections, there will be a huge challenge for health care workers (HCWs) to combat both viruses, MERS$\mathrm{CoV}$ and SARS-CoV-2. The current MERS-CoV case definition by Saudi CDC is highlighted in Table 3. 


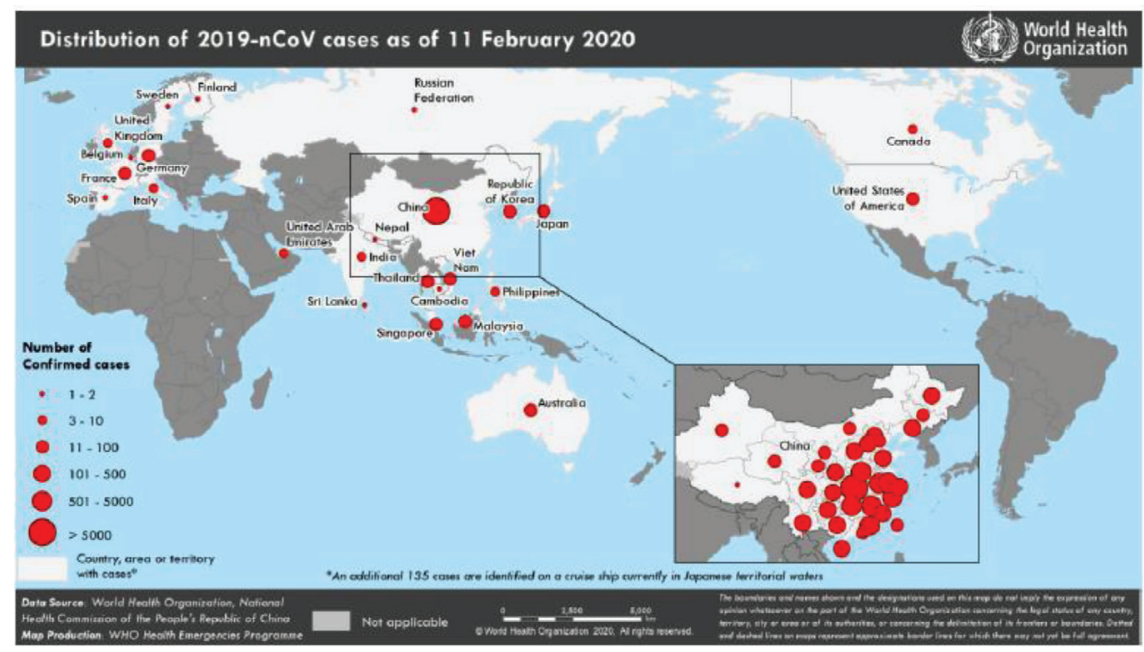

Figure 1 Countries, territories, or areas with reported confirmed cases of 2019-nCoV, February 11, 2020.

Table 1 Chinese CDC epidemiological and clinical criteria definitions

Epidemiological link to confirmed case or a part of symptomatic cluster and any two of

\begin{tabular}{l} 
Clinical criteria \\
1. Fever \\
2. Existence of pneumonia on chest X-ray \\
3. Normal or decreased white blood cell count \\
4. Decreased lymphocyte count \\
\hline
\end{tabular}

Table 2 WHO suspected case definition for COVID-19

A. Patient with severe acute respiratory infection (fever, cough and requiring admission to the hospital) AND there is no other etiology that fully explains the clinical presentation AND a history of travel to or residence in China during the 14 days prior to symptoms onset

B. Patient with any acute respiratory illness AND at least one of the following during the 14 days prior to symptoms onset:

1. Contact with a confirmed or probable case of COVID-19 infection or

2. Worked in or attended a health-care facility where patients with confirmed or probable COVID-19 acute respiratory disease patients were being treated

Table 3 MERS-CoV case definition for suspected case as per the command and control center

\section{Clinical criteria}

A. Severe pneumonia (severity score $\geq 3$ points) or ARDS (based on clinical or radiological evidence) ${ }^{x}$

B. Unexplained deterioration of a chronic condition of patients with congestive heart failure or chronic kidney disease on hemodialysis*

C. Acute febrile illness $\left(T \geq 38^{\circ} \mathrm{C}\right)$ with/without respiratory symptoms ${ }^{* *}$

D. Gastrointestinal symptoms (diarrhea or vomiting) AND leukopenia (white blood cell $\leq 3.5 \times 10^{9} / \mathrm{L}$ ) OR thrombocytopenia (platelets $<150 \times 10^{9} / \mathrm{L}$ )

Epidemiological link any of the following:

1. Exposure to a confirmed case of MERS-CoV infection

2. Visit to a health-care facility where MERS-CoV patient(s) has recently (within 2 weeks) been identified/treated

3. Contact with dromedary camels or consumption of camel products (e.g., raw meat, unpasteurized milk, urine)

"In adults and does not need an epidemiological criterion. "In adult and children and both need an epidemiological link within 14 days before symptoms onset. ARDS, Acute Repiratory Distress Syndrome.
After 7 years of MERS-CoV circulation in KSA, intensive efforts by the Saudi Ministry of Health $(\mathrm{MoH})$ have led to drastic improvement in infection control practices in Health-Care Facilities (HCFs) kingdom wide and most health-care workforce having a very high index of suspicion for any suspected MERS-CoV cases, especially at HCF's emergency rooms and hemodialysis units across the kingdom.

But with the lack of standardized and approved point of care testing for MERS-CoV, HCWs continue to be challenged with rapidly detecting and properly isolating patients with MERS-CoV, especially among patients with congestive heart failure $(\mathrm{CHF})$ and chronic kidney disease (CKD) who present with unexplained deterioration of their chronic conditions. This challenge will now be faced by the whole global health community dealing with COVID-19 since the disease tends to be more prevalent and more severe in patients with $\mathrm{CHF}$ or $\mathrm{CKD}$. Those patients can present with broad range of signs and symptoms and should now be tested for both MERS-CoV and SARS-CoV-2 simultaneously, and with the already wide international spread of SARS-CoV-2, the travel history to China in the last 14 days should be ignored.

The new COVID-19 guidance document [12] produced by Saudi CDC provides HCFs with a new visual triage (VT) for ARI that has a checklist for placing patients in isolation precautions with score $\geq 6$, with the weight of the points to history of travel to China in the last 14 days prior to symptoms onset, which is given five points. This contrasts with the MERS-CoV MoH guiding document [13] in which VT for ARI requires a score of $\geq 4$ to place patients in isolation. None of these VT scores have been validated to show effectiveness.

With the ongoing MERS-CoV circulation in animal reservoir (dromedary camels) in the Arabian Peninsula and the continuing zoonotic spillover with $70 \%$ of cases resulting from nosocomial transmission [14], the risk of emergence of COVID-19 within the same community would be overwhelming to the health-care infrastructure of the countries affected by MERS-CoV. This is a true concern with the already rapid international spread to 25 countries outside China, especially with the ability of SARS-CoV-2 to spread from a totally asymptomatic person [15].

Since the declaration of PHEIC by WHO on January 31, 2020 [16], 16 cities in mainland China with a population of around 60 million people remain under quarantine [17]. Many countries including Japan, the United States, France, Australia, Sri Lanka, Germany, 
Saudi Arabia, Russia, and Thailand among others evacuated their citizens through chartered flights [18], and several countries have recommended travelers to avoid nonessential travel to China and cancelled scheduled flight in and out of China [19]. Many countries [12] enforced public health measures at ports of entry with trained staff, appropriate space, and equipment and supplies for managing potential ill travelers, implementing entry screening including temperature recording for travelers arriving from China (directly or indirectly), and mandatory declaration of recent travel to china to immigration and passport officers. Although these measures may prevent the entry of active COVID-19 cases, history taught us that this system is not fully effective in preventing virus introduction into a country.

As per Saudi CDC, other public health measures include obligatory reporting of HCFs by calling a specific number and through the Health Electronic Surveillance Network. This would activate a rapid response team tasked with completing epidemiological investigation in HCFs and community spontaneously, categorizing household and community contacts as per their symptoms, which, as with MERS-CoV, underplays the need for more liberal testing or isolating asymptomatic contacts [20].

A comparative analysis of factors influencing MERS-CoV outbreak in KSA compared to South Korea identified several exacerbating factors: which includes poor access to health-care professionals at general practitioner level, which can result in delays between disease development, progression, and diagnosis, individuals who relied on camels for daily living denied the relation between camels and MERSCoV: kicking off the "Kiss Your Camel campaign," which became increasingly popular in Social Media in 2015, and 75\% of HCWs were unaware of how disease spreads, with a general feeling of workers and citizens is largely that MERS was not a substantial problem [21]. Similarly, the current COVID-19 outbreak and response have been accompanied by a massive "infodemic" - an overabundance of information-some accurate and many not-that makes it hard for people to find trustworthy sources and reliable guidance [10], as most turn to social media, it is vital to track and respond to myths and rumors, an up-to-date reference should be maintained.

\section{CONFLICTS OF INTEREST}

The authors declare they have no conflicts of interest.

\section{AUTHORS' CONTRIBUTION}

The authors contributed equally to the article.

\section{REFERENCES}

[1] Zaki AM, van Boheemen S, Bestebroer TM, Osterhaus ADME, Fouchier RAM. Isolation of a novel coronavirus from a man with pneumonia in Saudi Arabia. N Engl J Med 2012;367;1814-20.

[2] World Health Organization (WHO). Middle East respiratory syndrome coronavirus (MERS-CoV), MERS situation Update November 2019. WHO; 2020. Available from: www.who.int/ emergencies/mers-cov/en/ (accessed February 16, 2020).

[3] Ministry of Health (MOH). Command and Control Center. National Public Health Events, 2020. Ministry of Health; 2020.
Available from: https://www.moh.gov.sa/en/CCC/Pages/default. aspx (accessed February 16, 2020)

[4] Assiri A, McGeer A, Perl TM, Price CS, Al Rabeeah AA, Cummings DAT, et al. Hospital outbreak of middle east respiratory syndrome coronavirus. N Engl J Med 2013;369;407-16.

[5] Oboho IK, Tomczyk SM, Al-Asmari AM, Banjar AA, Al-Mugti H, Aloraini MS, et al. 2014 MERS-CoV outbreak in Jeddah - a link to health care facilities. N Engl J Med 2015;372;846-54.

[6] Fagbo SF, Skakni L, Chu DKW, Garbati MA, Joseph M, Peiris M, et al. Molecular epidemiology of hospital outbreak of middle east respiratory syndrome, Riyadh, Saudi Arabia, 2014. Emerg Infect Dis 2015;21;1981-88.

[7] Alenazi TH, Al Arbash H, El-Saed A, Alshamrani MM, BaffoeBonnie $\mathrm{H}$, Arabi YM, et al. Identified transmission dynamics of middle east respiratory syndrome coronavirus infection during an outbreak: implications of an overcrowded emergency department. Clin Infect Dis 2017;65;675-9.

[8] Huang C, Wang Y, Li X, Ren L, Zhao J, Hu Y, et al. Clinical features of patients infected with 2019 novel coronavirus in Wuhan, China. Lancet 2020;395;497-506.

[9] Zhu N, Zhang D, Wang W, Li X, Yang B, Song J, et al. A novel coronavirus from patients with pneumonia in China, 2019. N Engl J Med 2020;382;727-33.

[10] World Health Organization. Novel Coronavirus (2019-nCoV) Situation Report - 22. Geneva, Switzerland: World Health Organization; 2020.

[11] World Health Organization. Global Surveillance for human infection with novel coronavirus (2019-nCoV): Interim guidance v3. Geneva, Switzerland: World Health Organization; 2020. Available from: WHO/2019-nCoV/SurveillanceGuidance/2020.3

[12] Saudi Center for Disease prevention and Control. Novel Corona Virus (2019-nCoV) Infection Guidelines V1.0. Kingdom of Saudi Arabia: Saudi Center for Disease Prevention and Control Ministry of Health; 2020.

[13] Command and Control Center. Middle East Respiratory Syndrome Coronavirus; Guidelines for Healthcare Professionals, 2018, v5.1. Kingdom of Saudi Arabia: Ministry of Health; 2018.

[14] Munster VJ, Koopmans M, van Doremalen N, van Riel D, de Wit E. A novel coronavirus emerging in China - key questions for impact assessment. N Engl J Med 2020;382;692-94.

[15] Rothe C, Schunk M, Sothmann P, Bretzel G, Froeschl G, Wallrauch C, et al. Transmission of 2019-nCoV infection from an asymptomatic contact in Germany. N Engl J Med 2020.

[16] World Health Organization. Disease outbreak news (DONs). Geneva, Switzerland: World Health Organization; 2020. Available from: https://www.who.int/csr/don/en/.

[17] Griffiths J, Woodyatt A. China goes into emergency mode as number of confirmed Wuhan coronavirus cases reaches 2,700. CNN Updated 1648 GMT (0048 HKT); 2020.

[18] Jiang S, Stracqualursi V. US arranging charter flight to evacuate American diplomats and citizens out of China amid coronavirus outbreak, official says. CNN Updated 1827 GMT (0227 HKT); 2020.

[19] Centers for Disease Control and Prevention. Novel Coronavirus in China. CDC; 2020. https://wwwnc.cdc.gov/travel/notices/ warning/novel-coronavirus-china

[20] Memish ZA. Call to action for improved case definition and contact tracing for MERS-CoV. J Travel Med 2019;26;pii: taz001.

[21] Willman M, Kobasa D, Kindrachuk J. A Comparative analysis of factors influencing two outbreaks of Middle Eastern respiratory syndrome (MERS) in Saudi Arabia and South Korea. Viruses 2019;11;1119. 